AperTO - Archivio Istituzionale Open Access dell'Università di Torino

\title{
Generalized B-splines in isogeometric analysis
}

\section{This is the author's manuscript}

Original Citation:

\section{Availability:}

This version is available http://hdl.handle.net/2318/1714814

since 2019-10-30T17:25:40Z

Publisher:

Springer New York LLC

Published version:

DOI:10.1007/978-3-319-59912-0_12

Terms of use:

Open Access

Anyone can freely access the full text of works made available as "Open Access". Works made available under a Creative Commons license can be used according to the terms and conditions of said license. Use of all other works requires consent of the right holder (author or publisher) if not exempted from copyright protection by the applicable law. 


\title{
Generalized B-splines in Isogeometric Analysis
}

Carla Manni, Fabio Roman and Hendrik Speleers

\begin{abstract}
In this paper we survey the use of generalized B-splines in isogeometric Galerkin and collocation methods. Generalized B-splines are a special class of Tchebycheffian B-splines, and form an attractive alternative to standard polynomial B-splines and NURBS in both modeling and simulation. We summarize their definition and main properties, and we illustrate their use in a selection of numerical examples in the context of isogeometric analysis. For their interest in practical applications, we mainly focus on trigonometric and hyperbolic generalized B-splines.
\end{abstract}

\section{Introduction}

Isogeometric Analysis ( $\operatorname{IgA})$ is a technology introduced nearly a decade ago in a seminal paper by Hughes et al. [25] that unifies Computer Aided Design (CAD) and Finite Element Analysis (FEA). CAD software, used in industry for geometric modeling, typically describes physical domains by means of tensor-product B-splines or Non-Uniform Rational B-Splines (NURBS). Such geometries are then further processed in the analysis phase. One of the key concepts in IgA is to use the same discretization and representation tools for the design as well as for the analysis (in an isoparametric environment), providing a true design-through-analysis methodology $[14,25]$. The isogeometric approach based on B-splines/NURBS shows im-

Carla Manni

Department of Mathematics, University of Rome "Tor Vergata", Via della Ricerca Scientifica, 00133 Rome, Italy, e-mail: manni@mat.uniroma2.it

Fabio Roman

Department of Mathematics, University of Turin, Via Carlo Alberto 10, 10123 Turin, Italy, e-mail: fabio.roman@unito.it

Hendrik Speleers

Department of Mathematics, University of Rome "Tor Vergata", Via della Ricerca Scientifica, 00133 Rome, Italy, e-mail: speleers@mat.uniroma2.it 
portant advantages over standard finite element approaches. The geometry of the physical domain is exactly described, so the interaction with the CAD system during any further refinement process in the analysis phase is eliminated. In addition, B-spline and NURBS spaces possess an inherent higher smoothness than those in standard FEA, leading to a higher accuracy per degree of freedom. The concept of IgA leads to improved convergence and smoothness properties of the PDE solutions and faster overall simulations. Thanks to the successful application in various engineering fields (from structural mechanics to fluid-structure interaction), IgA is rapidly becoming a mainstream analysis methodology and a new paradigm for geometric design.

Even though NURBS are the de facto standard in CAD systems [39], they suffer from a few major drawbacks. For example, they lack an exact description of transcendental curves of interest in applications, and their parameterization of conics does not correspond to the arc length. In addition, NURBS behave poorly with respect to differentiation and integration, which are crucial operators in analysis. On the other hand, in the literature there exist alternatives to overcome such problems as we will briefly review in the following.

Standard polynomial splines of degree $p$ are smooth functions belonging piecewisely to the space $\mathbb{P}_{p}$ of algebraic polynomials of degree less than or equal to $p$. This concept can be generalized in two directions, see [44, Chapter 11]: (a) the spaces the functions belong to piecewisely can be more general than algebraic polynomials, and (b) the derivative conditions defining smoothness at each knot can be replaced by more general linear functionals. Some special cases of this wide generalizations have been independently studied by several authors primarily as tools in Computer Aided Geometric Design (CAGD).

Extended Tchebycheff (ET-) spaces of dimension $p+1$ on a given interval $J$ are spaces such that any non-trivial element has at most $p$ zeros (counting multiplicity) in $J$; these are a natural generalization of algebraic polynomial spaces. Similarly, Tchebycheffian splines, i.e., smooth functions belonging piecewisely to ET-spaces, are a natural and elegant generalization of polynomial splines, see [44, Chapter 9]. They offer several advantages over standard (algebraic) polynomial splines, mainly due to the wide variety of ET-spaces. Despite this flexibility, many results of the polynomial framework extend in an elegant way to the broader Tchebycheff framework. As it is difficult to trace all the works on Tchebycheffian splines, we limit ourselves to mention [26] for locally supported bases, [37] for a blossoming approach and [30] for a knot insertion procedure. We refer the reader to [44] for an extended bibliography on the topic.

In this paper we focus on a special class of Tchebycheffian splines, the so-called generalized splines. According to [28], with this term we denote smooth functions belonging piecewisely to spaces of the form $\left\langle 1, x, \ldots, x^{p-2}, \bar{U}(x), \bar{V}(x)\right\rangle$, with suitable assumptions on the functions $\bar{U}, \bar{V}$ and usual derivative conditions defining smoothness at each knot. Therefore, the generalized splines considered here are less general than those described in [44, Chapter 11]. They can be seen as the minimal extension of standard polynomial splines still offering a wide variety of additional flexibility in geometric modeling (see, e.g., [11, 12, 27, 28, 31, 38]) and numerical 
simulation (see, e.g., [32, 33, 35]). This good compromise between "minimal distance" from the polynomial case and flexibility is the motivation of our choice. Generalized splines possess many fundamental features of standard polynomial splines. In particular, they admit a representation in terms of basis functions with similar properties to polynomial B-splines. Such basis functions are referred to as Generalized B-splines (GB-splines).

GB-splines with a suitable selection of their section spaces - typically including polynomial and hyperbolic/trigonometric functions - allow for an exact representation of polynomial curves, conics, helices and other profiles of salient interest in applications. In particular, conics are well parameterized by hyperbolic/trigonometric GB-splines. Moreover, in contrast to NURBS, they behave completely similar to $\mathrm{B}$-splines with respect to differentiation and integration. Thanks to their structural similarity to polynomial B-splines, GB-splines are plug-to-plug compatible with Bsplines in modeling and simulation software.

In this paper we survey some recent results on the use of GB-splines as bases for the discretization spaces in numerical simulation according to the isogeometric paradigm. The goal of our survey is twofold. First, we want to highlight the structural similarity between B-splines and GB-splines, and illustrate this by means of their behavior with respect to local refinement (see $[8,10,34])$ and the spectral properties of the matrices obtained in Galerkin/collocation discretizations (see [42]). Second, we show how a fine-tuning of the section spaces - according to a problemoriented strategy taking into account the geometrical and/or analytical peculiar issues of the specific addressed problem - generally results in a gain from the accuracy point of view, see [32, 33, 34, 35].

The remainder of the paper is divided into 4 sections. Section 2 presents the definition and main properties of GB-splines. A short summary of isogeometric Galerkin and collocation methods is provided in Section 3. Section 4 compares numerical discretizations based on B-splines and GB-splines according to the isogeometric paradigm for three model differential problems taken from the literature. Finally, Section 5 collects some concluding remarks.

\section{Generalized B-splines}

In this section we present the definition and main properties of GB-splines. We start by defining the almost-polynomial spaces we are interested in.

\subsection{Almost-polynomial spaces}

Let $\bar{U}, \bar{V}$ be two univariate functions in $C^{p}([a, b])$ such that the space spanned by their $(p-1)$-th derivatives $\left\langle D^{(p-1)} \bar{U}, D^{(p-1)} \bar{V}\right\rangle$ is an ET-space of dimension 2 on $[a, b]$, i.e., any non-trivial element in the space has at most one zero (including mul- 
tiplicity) in $[a, b]$. Then, we consider the following almost-polynomial space:

$$
\mathbb{P}_{p}^{\bar{U}, \bar{V}}([a, b]):=\left\langle 1, x, \ldots, x^{p-2}, \bar{U}(x), \bar{V}(x)\right\rangle, \quad x \in[a, b] .
$$

The functions $\bar{U}, \bar{V}$ can be carefully selected in order to incorporate specific features in the space (1). Popular choices are:

$$
\begin{array}{ll}
\mathbb{P}_{p}([a, b]):=\left\langle 1, x, \ldots, x^{p-2}, x^{p-1}, x^{p}\right\rangle, & \\
\mathbb{H}_{p, \alpha}([a, b]):=\left\langle 1, x, \ldots, x^{p-2}, \cosh (\alpha x), \sinh (\alpha x)\right\rangle, & 0<\alpha \in \mathbb{R}, \\
\mathbb{T}_{p, \alpha}([a, b]):=\left\langle 1, x, \ldots, x^{p-2}, \cos (\alpha x), \sin (\alpha x)\right\rangle, & 0<\alpha(b-a)<\pi .
\end{array}
$$

We refer to [13] for a detailed analysis of the properties of spaces of the form (1). In particular, they are ET-spaces of dimension $p+1$ and admit a basis which is a natural generalization of standard Bernstein polynomials. Moreover, for fixed values of the involved parameters, the spaces (3) and (4) have the same approximation power as the polynomial space in (2), see [13, Section 3]. The spaces (3) and (4) have been widely used in geometric modeling and constrained interpolation/approximation because the phase parameters $\alpha$ can be exploited as shape-parameters [27, 31, 48].

In the following we are interested in spaces of smooth piecewise functions with sections in spaces of the form (1). Therefore, the space (1) will be referred to as section space. In the next section we describe how to build basis functions for such piecewise spaces that possess the same nice properties as standard polynomial Bsplines.

\subsection{GB-splines: definition and properties}

B-splines are the most famous basis for the space of polynomial splines. They can be defined in different ways, see [5,44], but probably their most popular definition is given in terms of a recurrence relation. For $n \geq 1$ and $p \geq 0$, let $\Xi:=\left\{\xi_{i}\right\}_{i=1}^{n+2 p+1}$ be a non-decreasing sequence of knots. Without loss of generality, we assume $\Xi$ to be open, i.e., its end knots have a multiplicity $p+1$,

$$
\xi_{1}=\cdots=\xi_{p+1}<\cdots \leq \xi_{i} \leq \xi_{i+1} \leq \cdots<\xi_{n+p+1}=\cdots=\xi_{n+2 p+1} .
$$

The $i$-th B-spline $B_{i, p, \Xi}$ of degree $p$ over the knot sequence $\Xi$ is defined recursively as follows. For $p=0$,

$$
B_{i, 0, \Xi}(x):= \begin{cases}1, & \text { if } x \in\left[\xi_{i}, \xi_{i+1}\right) \\ 0, & \text { elsewhere, }\end{cases}
$$

and for $p \geq 1$, 


$$
B_{i, p, \Xi}(x):=\frac{x-\xi_{i}}{\xi_{i+p}-\xi_{i}} B_{i, p-1, \Xi}(x)+\frac{\xi_{i+1+p}-x}{\xi_{i+1+p}-\xi_{i+1}} B_{i+1, p-1, \Xi}(x),
$$

where fractions with zero denominators are considered to be zero. The B-spline $B_{i, p, \Xi}$ is a non-negative piecewise polynomial supported on the interval $\left[\xi_{i}, \xi_{i+p+1}\right]$. Moreover, it has a very elegant differentiation formula for $p \geq 1$,

$$
D_{+} B_{i, p, \Xi}(x)=p\left(\frac{B_{i, p-1, \Xi}(x)}{\xi_{i+p}-\xi_{i}}-\frac{B_{i+1, p-1, \Xi}(x)}{\xi_{i+p+1}-\xi_{i+1}}\right),
$$

and integration formula,

$$
\delta_{i, p, \Xi}:=\int_{\xi_{i}}^{\xi_{i+p+1}} B_{i, p, \Xi}(s) \mathrm{d} s=\frac{\xi_{i+p+1}-\xi_{i}}{p+1} .
$$

By combining the above formulas we arrive at an alternative recurrence relation for B-splines of degree $p \geq 1$ :

$$
B_{i, p, \Xi}(x)=\int_{\xi_{i}}^{x} \frac{B_{i, p-1, \Xi}(s)}{\delta_{i, p-1, \Xi}} \mathrm{d} s-\int_{\xi_{i+1}}^{x} \frac{B_{i+1, p-1, \Xi}(s)}{\delta_{i+1, p-1, \Xi}} \mathrm{d} s,
$$

where we use the convention that if $\delta_{i, k, \Xi}=0$ then

$$
\int_{\xi_{i}}^{x} \frac{B_{i, k, \Xi}(s)}{\delta_{i, k, \Xi}} \mathrm{d} s:= \begin{cases}1, & \text { if } x \geq \xi_{i+k+1} \\ 0, & \text { otherwise. }\end{cases}
$$

A generalization of (6) leads to B-spline-like functions with sections in spaces of the form (1), see [28, 36] and references therein. They will be called GB-splines of degree $p$. Note that the term degree is used here to stress the similarity with the polynomial case. Given a knot sequence $\Xi$ as in (5), we select for each $\xi_{i}<\xi_{i+1}$ an almost-polynomial space $\mathbb{P}_{p}^{\bar{U}_{i}, \bar{V}_{i}}\left(\left[\xi_{i}, \xi_{i+1}\right]\right)$ generated by the functions $\bar{U}_{i}, \bar{V}_{i}$. We denote by $\bar{u}_{i}, \bar{v}_{i}$ the unique elements in $\left\langle D^{(p-1)} \bar{U}_{i}, D^{(p-1)} \bar{V}_{i}\right\rangle$ satisfying

$$
\bar{u}_{i}\left(\xi_{i}\right)=1, \quad \bar{u}_{i}\left(\xi_{i+1}\right)=0, \quad \bar{v}_{i}\left(\xi_{i}\right)=0, \quad \bar{v}_{i}\left(\xi_{i+1}\right)=1 .
$$

For notational convenience, we also define the piecewise functions $U, V$ by

$$
U(x):=\bar{U}_{i}(x), \quad V(x):=\bar{V}_{i}(x), \quad x \in\left[\xi_{i}, \xi_{i+1}\right) .
$$

Due to the structure of spaces of the form (1), it is natural to consider a recurrence relation starting from GB-splines of degree 1.

Definition 1. Given a knot sequence $\Xi$ and a sequence of almost-polynomial spaces $\mathbb{P}_{p}^{\bar{U}_{i}, \bar{V}_{i}}\left(\left[\xi_{i}, \xi_{i+1}\right]\right)$, the $i$-th GB-spline $B_{i, p, \Xi}^{U, V}$ of degree $p$ over $\Xi$ is defined recursively as follows. For $p=1$, 

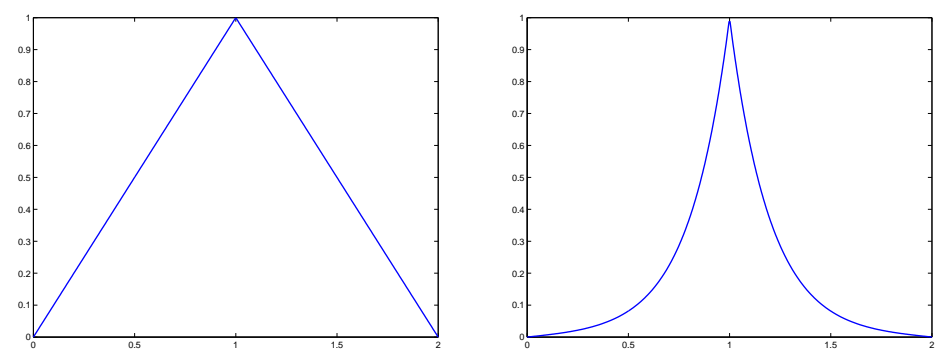

Fig. 1 GB-spline of degree 1 with knot sequence $\Xi=\{0,1,2\}$. Left: The standard polynomial case. Right: The hyperbolic case with $\alpha_{i}=5$.

$$
B_{i, 1, \Xi}^{U, V}(x):= \begin{cases}\bar{v}_{i}(x), & \text { if } x \in\left[\xi_{i}, \xi_{i+1}\right) \\ \bar{u}_{i+1}(x), & \text { if } x \in\left[\xi_{i+1}, \xi_{i+2}\right) \\ 0, & \text { elsewhere }\end{cases}
$$

and for $p \geq 2$,

$$
B_{i, p, \Xi}^{U, V}(x):=\int_{\xi_{i}}^{x} \frac{B_{i, p-1, \Xi}^{U, V}(s)}{\delta_{i, p-1, \Xi}^{U, V}} \mathrm{~d} s-\int_{\xi_{i+1}}^{x} \frac{B_{i+1, p-1, \Xi}^{U, V}(s)}{\delta_{i+1, p-1, \Xi}^{U, V}} \mathrm{~d} s,
$$

where

$$
\delta_{i, p, \Xi}^{U, V}:=\int_{\xi_{i}}^{\xi_{i+p+1}} B_{i, p, \Xi}^{U, V}(s) \mathrm{d} s,
$$

and we use the convention that if $\delta_{i, k, \Xi}^{U, V}=0$ then

$$
\int_{\xi_{i}}^{x} \frac{B_{i, k, \Xi}^{U, V}(s)}{\delta_{i, k, \Xi}^{U, V}} \mathrm{~d} s:= \begin{cases}1, & \text { if } x \geq \xi_{i+k+1}, \\ 0, & \text { otherwise. }\end{cases}
$$

GB-splines with only section spaces in (2), (3) and (4) will be referred to as standard B-splines, hyperbolic (or exponential) GB-splines and trigonometric GBsplines, respectively. Two GB-splines of degree 1 are depicted in Figure 1, and some sets of cubic GB-splines are illustrated in Figure 2.

GB-splines share many fundamental features with standard B-splines (see, e.g., $[28,48])$. We collect some properties in the next proposition.

Proposition 1. Let $\left\{B_{i, p, \Xi}^{U, V}: i=1, \ldots, n+p\right\}$ be a set of GB-splines of degree $p \geq 2$ over the knot sequence $\stackrel{\Xi}{\Xi}$. The following properties hold:

- Piecewise structure: $B_{i, p, \Xi}^{U, V} \in \mathbb{P}_{p}^{\bar{U}_{m}, \bar{V}_{m}}\left(\left[\xi_{m}, \xi_{m+1}\right)\right)$;

- Positivity: $B_{i, p, \Xi}^{U, V}(x)>0, x \in\left(\xi_{i}, \xi_{i+p+1}\right)$;

- Local support: $B_{i, p, \Xi}^{U, V}(x)=0, x \notin\left[\xi_{i}, \xi_{i+p+1}\right]$; 

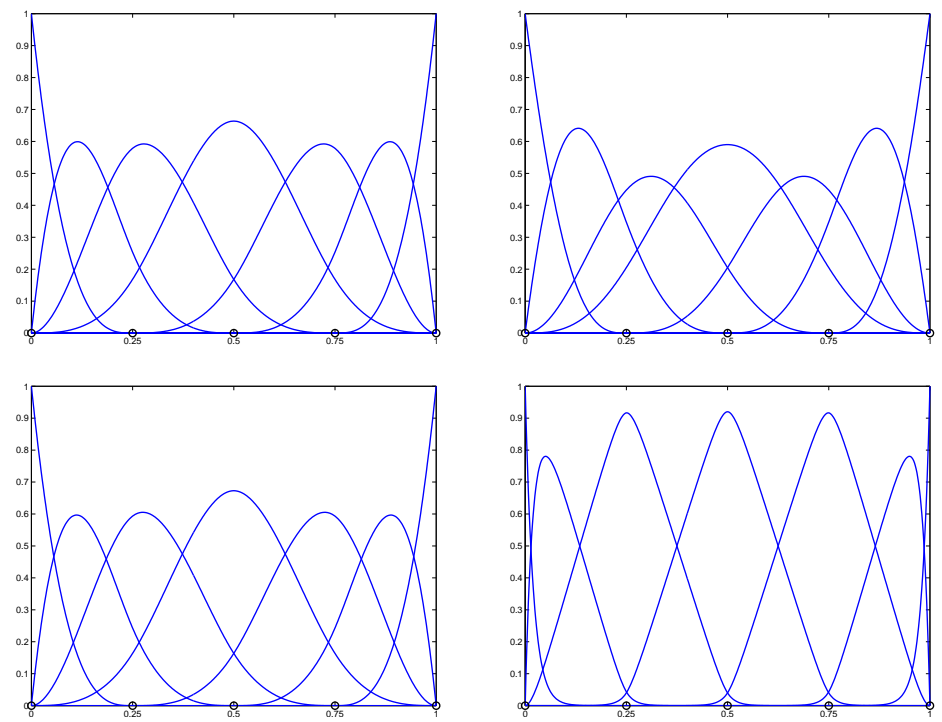

Fig. 2 Examples of GB-splines of degree 3 defined on the knot sequence $\Xi=$ $\{0,0,0,0,1 / 4,1 / 2,3 / 4,1,1,1,1\}$. Top: Trigonometric GB-splines with $\alpha_{i}=\frac{2}{3} \pi$ (left) and $\alpha_{i}=$ $3 \pi$ (right). Bottom: Hyperbolic GB-splines with $\alpha_{i}=3$ (left) and $\alpha_{i}=50$ (right).

- Smoothness: $B_{i, p, \Xi}^{U, V}$ is $p-\mu_{j}$ times continuously differentiable at $\xi_{j}$, being $\mu_{j}$ the multiplicity of $\xi_{j}$ in $\left\{\xi_{i}, \ldots, \xi_{i+p+1}\right\}$;

- Local partition of unity: $\sum_{i=m-p}^{m} B_{i, p, \Xi}^{U, V}(x)=1, x \in\left[\xi_{m}, \xi_{m+1}\right)$;

- Local linear independence: $\left\{B_{i, p, \Xi}^{U, V}: i=m-p, \ldots, m\right\}$ are linearly independent on $\left[\xi_{m}, \xi_{m+1}\right)$;

- Differentiation: $D_{+} B_{i, p, \Xi}^{U, V}(x)=\frac{B_{i, p-1, \Xi}^{U, V}(x)}{\delta_{i, p-1, \Xi}^{U, V}}-\frac{B_{i+1, p-1, \Xi}^{U, V}(x)}{\delta_{i+1, p-1, \Xi}^{U, V}}$.

In complete analogy with the polynomial case, there is also a knot insertion procedure available for GB-splines, see [30, 48].

Proposition 2. Let $\widehat{\Xi}$ be the knot sequence obtained by inserting a new knot $\hat{\xi}$ in the knot sequence $\Xi$ in the interval $\left(\xi_{p+1}, \xi_{n+p+1}\right)$, and set $\widehat{U}:=U, \widehat{V}:=V$. For fixed $i$, let $\mu_{j}$ be the multiplicity of $\xi_{j}$ in $\left\{\xi_{i}, \ldots, \xi_{i+p+1}\right\}$. Then, for $p \geq 2$ we have

$$
B_{i, p, \Xi}^{U, V}(x)=\widehat{\omega}_{i, p}^{\widehat{U}, \widehat{V}} B_{i, p, \widehat{U}, \widehat{\Xi}}^{\widehat{\Xi}}(x)+\widehat{\sigma}_{i, p}^{\widehat{U}, \widehat{V}} B_{i+1, p, \widehat{\Xi}, \widehat{\Xi}}^{\widehat{U}}(x),
$$

where

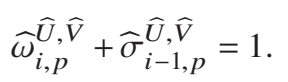

Moreover, if $\mu_{i}>1$ then 


$$
\widehat{\omega} \widehat{U}, \widehat{V}:= \begin{cases}1, & \text { if } \xi_{i+p} \leq \hat{\xi}, \\ \prod_{\ell=\mu_{i}-1}^{p-1} \frac{\delta_{i, \ell, \widehat{\Xi}}^{\widehat{U}, \widehat{\Xi}}}{\delta_{i, \ell, \Xi}^{U, V},} & \text { if } \xi_{i}<\hat{\xi}<\xi_{i+p}, \\ 0, & \text { if } \widehat{\xi} \leq \xi_{i},\end{cases}
$$

while, if $\mu_{i}=1$ then

$$
\widehat{\omega_{i, p}^{U}, \widehat{V}}:= \begin{cases}1, & \text { if } \xi_{i+p} \leq \hat{\xi}, \\ \frac{D_{+} B_{i, 1, \Xi}\left(\xi_{i}\right)}{D_{+} B_{i, 1, \widehat{\Xi}}\left(\xi_{i}\right)} \prod_{\ell=1}^{p-1} \frac{\delta_{i, \ell, \widehat{\Xi}}^{\widehat{U}, \widehat{V}}}{\delta_{i, \ell, \Xi}^{U, V}}, & \text { if } \xi_{i}<\hat{\xi}<\xi_{i+p}, \\ 0, & \text { if } \widehat{\xi} \leq \xi_{i},\end{cases}
$$

with the usual convention that a product is 1 if the lower index exceeds the upper index.

The expressions in (9) and (10) are obtained by comparing the first non-vanishing (right) derivative at $\xi_{i}$ of the left and the right term in (7). A similar expression for $\widehat{\sigma} \widehat{U}, \widehat{V}$ can be obtained by comparing the first non-vanishing (left) derivative at $\xi_{i+p+1}$ of the left and the right term in (7). The equality (8) can be deduced by the local partition of unity property.

The space spanned by a set of GB-splines defined over a fixed knot sequence will be referred to as generalized spline space. For notational convenience, we denote by $\mathbb{S}_{p, \Xi}, \mathbb{S}_{p, \Xi}^{\mathbb{H}_{\alpha}}$ and $\mathbb{S}_{p, \Xi}^{\mathbb{T}_{\alpha}}$ the spaces spanned by standard B-splines, hyperbolic and trigonometric GB-splines, respectively.

It is clear that hyperbolic and trigonometric GB-splines allow for an exact representation of conics as well as some transcendental curves (helix, cycloid, ...). They are very attractive from the geometrical point of view. Indeed, in contrast to NURBS, they are able to provide parameterizations of conics close to the arc length so that equally spaced points in the parameter domain correspond to almost equally spaced points on the described curve. Since the section spaces (1) may be chosen differently on each knot interval, GB-splines allow for an exact representation of profiles composed by a sequence of curve segments of different kind: arcs of ellipses, hyperbolas, polynomial curves, etc.

Furthermore, the differential operator acts on trigonometric and hyperbolic generalized splines in the same way as on standard polynomial splines. More precisely,

$$
D_{+} \mathbb{S}_{p, \Xi}=\mathbb{S}_{p-1, \Xi}, \quad D_{+} \mathbb{S}_{p, \Xi}^{\mathbb{H}_{\alpha}}=\mathbb{S}_{p-1, \Xi}^{\mathbb{H}_{\alpha}}, \quad D_{+} \mathbb{S}_{p, \Xi}^{\mathbb{T}_{\alpha}}=\mathbb{S}_{p-1, \Xi}^{\mathbb{T}_{\alpha}}, \quad p \geq 2 .
$$

NURBS do not possess a property like (11). This property makes the structural similarity between hyperbolic/trigonometric GB-splines and standard B-splines even stronger.

Remark 1. Hyperbolic/trigonometric GB-splines approach standard B-splines of the same degree and over the same knot sequence when the local phase parameters $\alpha$ approach 0 . The same is true if, for fixed values of $\alpha$, the length of each knot interval $\xi_{i+1}-\xi_{i}$ tends to zero. 


\subsection{Uniform GB-splines}

In many practical applications uniform knot sequences are of particular interest. As for the standard polynomial case, GB-splines with uniform knot sequences enjoy special properties. In this section we summarize some of them.

Given the almost-polynomial space $\mathbb{P}_{p}^{U, V}([0,1])$, we denote by $\bar{u}, \bar{v}$ the unique elements in $\left\langle D^{(p-1)} U, D^{(p-1)} V\right\rangle$ satisfying

$$
\bar{u}(0)=1, \quad \bar{u}(1)=0, \quad \bar{v}(0)=0, \quad \bar{v}(1)=1 .
$$

Definition 2. The (normalized) cardinal GB-spline of degree $p \geq 1$ over the uniform knot sequence $\{0,1, \ldots, p+1\}$ with sections belonging to integer translations of $\mathbb{P}_{p}^{U, V}([0,1])$ is denoted by $\phi_{p}^{U, V}$ and is defined recursively as follows. For $p=1$,

$$
\phi_{1}^{U, V}(t):=\frac{1}{\delta_{1}^{U, V}} \begin{cases}\bar{v}(t), & \text { if } t \in[0,1), \\ \bar{u}(t-1), & \text { if } t \in[1,2), \\ 0, & \text { elsewhere }\end{cases}
$$

where

$$
\delta_{1}^{U, V}:=\int_{0}^{1} \bar{v}(s) \mathrm{d} s+\int_{1}^{2} \bar{u}(s-1) \mathrm{d} s .
$$

For $p \geq 2$,

$$
\phi_{p}^{U, V}(t):=\int_{0}^{t}\left(\phi_{p-1}^{U, V}(s)-\phi_{p-1}^{U, V}(s-1)\right) \mathrm{d} s .
$$

If $\mathbb{P}_{p}^{U, V}([0,1])=\mathbb{P}_{p}([0,1])$ then the function defined in Definition 2 is the standard (polynomial) cardinal B-spline of degree $p$, denoted by $\phi_{p}$. In the next proposition we list some properties of cardinal GB-splines (see, e.g., [42, Section 3.1]).

Proposition 3. Let $\phi_{p}^{U, V}$ be the cardinal GB-spline of degree $p \geq 2$. The following properties hold:

- Smoothness: $\phi_{p}^{U, V} \in C^{p-1}$;

- Positivity: $\phi_{p}^{U, V}(t)>0, t \in(0, p+1)$;

- Local support: $\phi_{p}^{U, V}(t)=0, t \notin(0, p+1)$;

- Partition of unity: $\sum_{k=1}^{p} \phi_{p}^{U, V}(k)=1$;

- Differentiation: $D \phi_{p}^{U, V}(t)=\phi_{p-1}^{U, V}(t)-\phi_{p-1}^{U, V}(t-1)$;

- Integration: $\int_{0}^{p+1} \phi_{p}^{U, V}(s) \mathrm{d} s=1$;

- Convolution relation: $\phi_{p}^{U, V}(t)=\int_{0}^{1} \phi_{p-1}^{U, V}(t-s) \mathrm{d} s$.

The hyperbolic cardinal GB-spline is denoted by $\phi_{p}^{\mathbb{H}_{\alpha}}$ and is defined by taking $U(t):=\cosh (\alpha t)$ and $V(t):=\sinh (\alpha t)$. In this case, we have

$$
\bar{u}(t)=\frac{\sinh (\alpha(1-t))}{\sinh (\alpha)}, \quad \bar{v}(t)=\frac{\sinh (\alpha t)}{\sinh (\alpha)},
$$


satisfying (12). The trigonometric cardinal GB-spline is denoted by $\phi_{p}^{\mathbb{T}_{\alpha}}$ and is defined by taking $U(t):=\cos (\alpha t)$ and $V(t):=\sin (\alpha t)$. In this case, we have

$$
\bar{u}(t)=\frac{\sin (\alpha(1-t))}{\sin (\alpha)}, \quad \bar{v}(t)=\frac{\sin (\alpha t)}{\sin (\alpha)},
$$

satisfying (12).

We now focus on the uniform knot sequence

$$
\left\{\xi_{1}, \ldots, \xi_{n+2 p+1}\right\}=\{\underbrace{0, \ldots, 0}_{p+1}, \frac{1}{n}, \frac{2}{n}, \ldots, \frac{n-1}{n}, \underbrace{1, \ldots, 1}_{p+1}\},
$$

and consider the same almost-polynomial space (1) on each knot interval. The corresponding GB-splines of degree $p$ according to Definition 1 are compactly denoted by

$$
\left\{B_{i, p}^{U, V}: i=1, \ldots, n+p\right\},
$$

and their space is denoted by $\mathbb{S}_{n, p}^{U, V}$. It turns out that

$$
\mathbb{S}_{n, p}^{U, V}=\left\{f \in C^{p-1}([0,1]): f \in \mathbb{P}_{p}^{\bar{U}, \bar{V}}\left(\left[\frac{i}{n}, \frac{i+1}{n}\right)\right), i=0, \ldots, n-1\right\} .
$$

In particular, let

$$
\left\{B_{i, p}^{\mathbb{H}_{\alpha}}: i=1, \ldots, n+p\right\}, \quad\left\{B_{i, p}^{\mathbb{T}_{\alpha}}: i=1, \ldots, n+p\right\}
$$

be the sets of hyperbolic and trigonometric GB-splines of degree $p$ defined over the knot sequence (13) with sections in (3) and (4), respectively. Then, it is easy to check that for $i=p+1, \ldots, n$ and $p \geq 2$,

$$
B_{i, p}^{\mathbb{H}_{\alpha}}(x)=\phi_{p}^{\mathbb{H}_{\alpha} / n}(n x-i+p+1), \quad B_{i, p}^{\mathbb{T}_{\alpha}}(x)=\phi_{p}^{\mathbb{T}_{\alpha / n}}(n x-i+p+1),
$$

in a complete analogy with the polynomial case. Their spaces are denoted by $\mathbb{S}_{n, p}^{\mathbb{H}_{\alpha}}$ and $\mathbb{S}_{n, p}^{\mathbb{T}_{\alpha}}$, respectively, while $\mathbb{S}_{n, p}$ is the standard polynomial spline space over the knots (13).

Remark 2. A main issue in working with GB-splines is the construction of efficient algorithms for their evaluation. We refer to [43] for a discussion and the description of an approximation procedure in case of uniform knots based on cardinal B-splines.

\subsection{Tensor-products and beyond}

Multivariate versions of GB-splines can be straightforwardly obtained by the usual tensor-product approach (in $d$ dimensions): 


$$
B_{\boldsymbol{i}, \boldsymbol{p}, \boldsymbol{\Xi}}^{\boldsymbol{U}, \boldsymbol{V}}=B_{i_{1}, p_{1}, \Xi_{1}}^{U_{1}, V_{1}} \otimes B_{i_{2}, p_{2}, \Xi_{2}}^{U_{2}, V_{2}} \otimes \cdots \otimes B_{i_{d}, p_{d}, \Xi_{d}}^{U_{d}, V_{d}},
$$

where different knot sequences, degrees and section spaces can be considered in the different directions. The structural simplicity makes tensor-product splines a powerful tool for both modeling and simulation.

Adaptive local refinement is important in applications. Unfortunately, a simple tensor-product spline structure lacks adequate local refinement. This triggered the interest in alternative spline structures supporting local refinement still retaining a local tensor-product structure. On this concern, we mention (analysis-suitable) Tsplines [29, 46], hierarchical splines [19, 21, 22], and Locally Refined (LR-) splines [16]. All of them can be seen as special cases of polynomial splines over T-meshes $[15,45]$. In the more recent literature we also find some specific extensions to the generalized spline setting. For example, generalized T-splines [6, 7], hierarchical generalized splines [34] and generalized splines on T-meshes [8, 9, 10] have been addressed.

In this section we limit ourselves to the simple but effective approach of hierarchical/multilevel bases, and we outline the construction of hierarchical bases in terms of a hierarchy of tensor-product GB-splines, see [22, 34, 47].

Let $\widehat{\Omega}$ be a hyper-rectangle in $\mathbb{R}^{d}$. We consider a sequence of nested tensorproduct $d$-variate generalized spline spaces defined on $\widehat{\Omega}$,

$$
\mathbb{W}^{0} \subset \mathbb{W}^{1} \subset \mathbb{W}^{2} \subset \cdots
$$

Any element of $\mathbb{W}^{\ell}$ is a piecewise function defined over a partition of $\widehat{\Omega}$ consisting of hyper-rectangles, which will be called cells of level $\ell$. We denote by

$$
\mathcal{B}^{\ell}:=\left\{b_{i, \ell}: i=1, \ldots, \operatorname{dim}\left(\mathbb{W}^{\ell}\right)\right\}
$$

the tensor-product GB-spline basis of $\mathbb{W}^{\ell}$, where the knot sequences are assumed to be open in each direction. Finally, we consider a nested sequence of closed subsets of $\widehat{\Omega}$,

$$
\widehat{\Omega}=: \Omega^{0} \supseteq \Omega^{1} \supseteq \Omega^{2} \supseteq \cdots,
$$

where each $\Omega^{\ell}$ is the union of a selection of cells of level $\ell$. The set

$$
\mathbf{\Omega}_{N}:=\left\{\Omega^{0}, \Omega^{1}, \ldots, \Omega^{N-1}\right\}, \quad N \geq 1,
$$

will be referred to as a hierarchy of subsets of $\widehat{\Omega}$ of depth $N$.

Given a sequence of spaces and bases as in (18)-(19) and a hierarchy of subsets as in (21), we can now construct the corresponding set of so-called hierarchical basis functions as follows. We first take all the basis elements in $\mathcal{B}^{0}$. Then, we apply an iterative procedure which selects at each level $\ell$ all the basis functions obtained in the previous step whose support is not entirely contained in $\Omega^{\ell}$ and all the basis functions in $\mathcal{B}^{\ell}$ whose support is entirely contained in $\Omega^{\ell}$. More precisely, we state the following definition. 

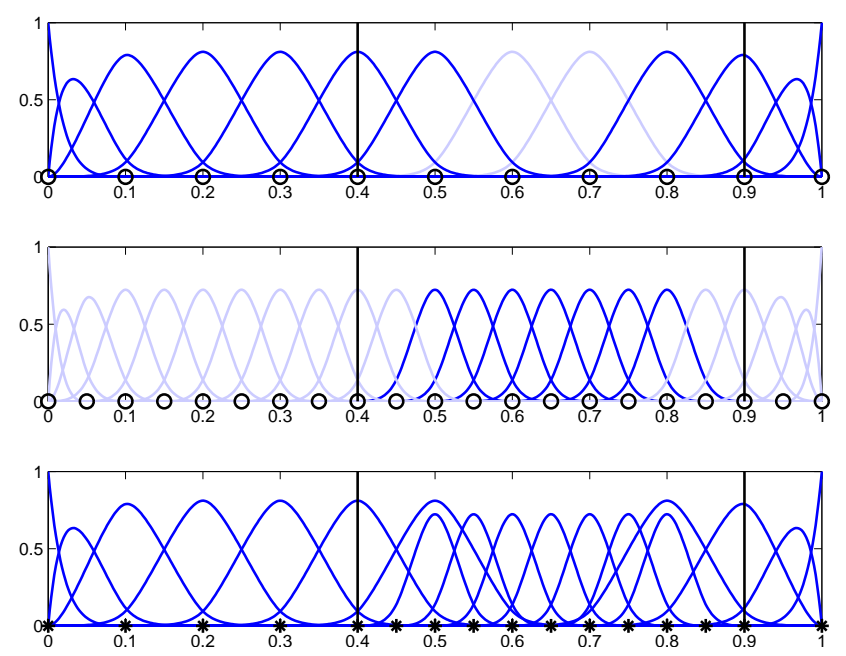

Fig. 3 Illustration of the construction of hierarchical cubic hyperbolic GB-splines with $\alpha=50$, $N=2, \Omega_{0}=[0,1], \Omega_{1}=[0.4,0.9]$. The light colored functions are removed in the construction of the hierarchical basis.

Definition 3. The hierarchical set of basis functions $\mathcal{H}_{\mathbf{\Omega}_{N}}$ associated with a hierarchy of subsets of $\widehat{\Omega}$ as in (21) is recursively constructed as follows:

i) $\mathcal{H}^{0}:=\left\{b_{i, 0} \in \mathcal{B}^{0}: \operatorname{supp}\left(b_{i, 0}\right) \neq \emptyset\right\}$;

ii) for $\ell=0, \ldots, N-2$ :

$$
\mathcal{H}^{\ell+1}:=\mathcal{H}_{C}^{\ell+1} \cup \mathcal{H}_{F}^{\ell+1},
$$

where

$$
\begin{aligned}
& \mathcal{H}_{C}^{\ell+1}:=\left\{b_{i, j} \in \mathcal{H}^{\ell}: \operatorname{supp}\left(b_{i, j}\right) \nsubseteq \Omega^{\ell+1}\right\}, \\
& \mathcal{H}_{F}^{\ell+1}:=\left\{b_{i, \ell+1} \in \mathcal{B}^{\ell+1}: \operatorname{supp}\left(b_{i, \ell+1}\right) \subseteq \Omega^{\ell+1}\right\} ;
\end{aligned}
$$

iii) $\mathcal{H}_{\mathbf{\Omega}_{N}}:=\mathcal{H}^{N-1}$.

The space $\mathbb{S}_{\boldsymbol{\Omega}_{N}}:=\left\langle b_{i, j}: b_{i, j} \in \mathcal{H}_{\boldsymbol{\Omega}_{N}}\right\rangle$ is called the hierarchical space associated with $\boldsymbol{\Omega}_{N}$. Since the elements in $\mathcal{B}^{\ell}$ are locally linearly independent, Definition 3 provides a set of linearly independent functions [22]. Therefore, the elements in $\mathcal{H}_{\boldsymbol{\Omega}_{N}}$ form a basis of $\mathbb{S}_{\boldsymbol{\Omega}_{N}}$, which will be referred to as the hierarchical basis of $\mathbb{S}_{\boldsymbol{\Omega}_{N}}$. Figure 3 shows the construction of the hierarchical basis for hierarchical cubic hyperbolic GB-splines on the interval $[0,1]$ associated with a hierarchy of depth 2.

The elements of the hierarchical basis are obviously non-negative but they do not form a partition of unity. Nevertheless, it is possible to construct an alternative basis of $\mathbb{S}_{\boldsymbol{\Omega}_{N}}$, the so-called truncated hierarchical basis whose elements form a convex partition of unity [22]. 
Remark 3. The standard definition of hierarchical bases and spaces summarized here assumes to deal with a sequence of nested linear spaces. However, Definition 3 also makes sense when the spaces $\mathbb{W}^{\ell}$ do not form a nested sequence. Giving up nestedness allows for more freedom in the construction and leads to the so-called multilevel bases [34]. Under the hypothesis of local linear independence for the basis functions considered in each space $\mathbb{W}^{\ell}$, the resulting set of multilevel functions are still linearly independent.

\section{Isogeometric Galerkin and collocation methods}

In this section we outline the Galerkin and collocation approximation of PDE solutions in the IgA framework. For the sake of simplicity, we illustrate the isogeometric approaches for second-order elliptic differential problems with homogeneous Dirichlet boundary conditions. A more comprehensive presentation can be found in [14].

Let $\mathcal{L}$ be a linear second-order elliptic differential operator on the domain $\Omega \subset \mathbb{R}^{d}$ with Lipschitz boundary $\partial \Omega$. We consider the differential problem

$$
\begin{cases}\mathcal{L} u=f, & \text { in } \Omega, \\ u=0, & \text { on } \partial \Omega\end{cases}
$$

for the unknown $u: \Omega \rightarrow \mathbb{R}$. This is the so-called strong form of the problem. The weak form of (22) is given by

$$
a(u, v)=F(v),
$$

where

$$
\begin{aligned}
& a: \mathbb{V} \times \mathbb{V} \rightarrow \mathbb{R} \text { is a bilinear form depending on } \mathcal{L}, \\
& F: \mathbb{V} \rightarrow \mathbb{R} \text { is a linear form depending on } f,
\end{aligned}
$$

and holds for any $v$ in the corresponding (homogeneous) solution space $\mathbb{V}_{0} \subset \mathbb{V}$.

Example 1. For the second-order differential problem with constant coefficients,

$$
\begin{cases}-\kappa \Delta u+\beta \cdot \nabla u+\gamma u=f, & \text { in } \Omega, \\ u=0, & \text { on } \partial \Omega\end{cases}
$$

we have $\mathbb{V}=H^{1}(\Omega), \mathbb{V}_{0}=H_{0}^{1}(\Omega)$ and

$$
a(u, v)=\int_{\Omega}(\kappa \nabla u \nabla v+(\boldsymbol{\beta} \cdot \nabla u+\gamma u) v), \quad F(v)=\int_{\Omega} f v .
$$




\subsection{Isogeometric Galerkin methods}

The Galerkin approach to approximate the solution of (22) is based on the weak form (23). First, we select a finite-dimensional approximation space on $\Omega$,

$$
\mathbb{V}_{h}:=\left\langle\varphi_{1}, \varphi_{2}, \ldots, \varphi_{m_{h}}\right\rangle \subset \mathbb{V}, \quad \operatorname{dim}\left(\mathbb{V}_{h}\right)=m_{h},
$$

related to a fineness parameter $h>0$, and

$$
\mathbb{V}_{h, 0}:=\left\{v_{h} \in \mathbb{V}_{h}: v_{h \mid \partial \Omega}=0\right\} \subset \mathbb{V}_{0}, \quad \operatorname{dim}\left(\mathbb{V}_{h, 0}\right)=n_{h}<m_{h}
$$

Then, we look for $u_{h}^{G} \in \mathbb{V}_{h, 0}$ as the solution of

$$
a\left(u_{h}^{G}, v_{h}\right)=F\left(v_{h}\right), \quad \forall v_{h} \in \mathbb{V}_{h, 0} .
$$

Assuming $\mathbb{V}_{h, 0}=\left\langle\varphi_{1}, \varphi_{2}, \ldots, \varphi_{n_{h}}\right\rangle$ and writing $u_{h}^{G}=\sum_{i=1}^{n_{h}} c_{i} \varphi_{i}$, condition (26) gives rise to a linear system $A \mathbf{c}=\mathbf{F}$, where

$A \in \mathbb{R}^{n_{h} \times n_{h}}$ is the stiffness matrix, $A_{i, j}:=a\left(\varphi_{j}, \varphi_{i}\right), i, j=1, \ldots, n_{h}$,

$\mathbf{F} \in \mathbb{R}^{n_{h}}$ is the load vector, $f_{i}:=F\left(\varphi_{i}\right), i=1, \ldots, n_{h}$.

Different Galerkin methods correspond to different choices of the subspace $\mathbb{V}_{h}$. Since the weak form (23) is expressed in terms of integrals (see Example 1), the construction of the stiffness matrix and load vector requires the use of numerical integration. Therefore, the efficiency of any Galerkin method deeply depends on the efficiency of the quadrature rules used in the construction of the corresponding linear systems.

In the standard formulation of $\operatorname{IgA}$, the physical domain $\Omega$ is represented by means of a global geometry map. We define the geometry map $\mathbf{G}$ from the parametric domain $\widehat{\Omega}:=[0,1]^{d}$ to the closure of $\Omega$ as (see Figure 4 )

$$
\mathbf{G}(\hat{\mathbf{x}}):=\sum_{i=1}^{m_{h}} \mathbf{P}_{i} \hat{\varphi}_{i}(\hat{\mathbf{x}}), \quad \mathbf{P}_{i} \in \mathbb{R}^{d}, \quad \hat{\mathbf{x}} \in \widehat{\Omega},
$$

where the basis functions

$$
\left\{\hat{\varphi}_{1}, \ldots, \hat{\varphi}_{m_{h}}\right\}
$$

have to be selected so as to produce an exact representation of the geometry. Following the isoparametric approach, the space $\mathbb{V}_{h}$ in (24) is then defined as

$$
\varphi_{i}(\mathbf{x}):=\hat{\varphi}_{i} \circ \mathbf{G}^{-1}(\mathbf{x})=\hat{\varphi}_{i}(\hat{\mathbf{x}}), \quad i=1, \ldots, m_{h}, \quad \mathbf{x}=\mathbf{G}(\hat{\mathbf{x}}) .
$$

Usually, the functions in (28) are chosen to be tensor-product B-splines or NURBS. In this paper we consider GB-splines whose section spaces will be selected according to a problem-oriented strategy.

The Galerkin formulation has been intensively and successfully employed in the isogeometric context. In contrast to the finite element context, where elementwise 

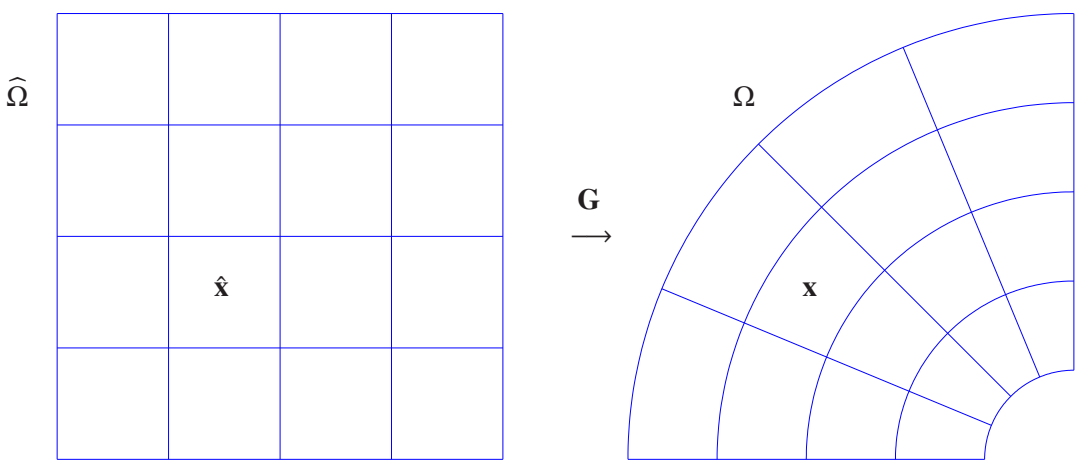

Fig. 4 Isogeometric Galerkin. Description of the physical domain $\Omega$ by means of a global geometry map $\mathbf{G}$.
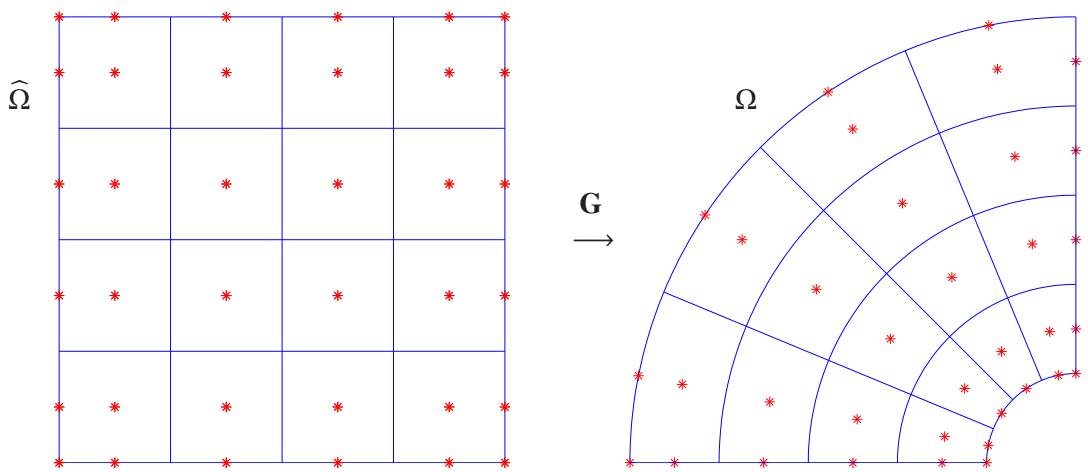

Fig. 5 Isogeometric collocation. Description of the physical domain $\Omega$ by means of a global geometry map $\mathbf{G}$ and related collocation points.

Gauss quadrature is known to be optimal, it is not yet completely clear how to construct efficient IgA quadrature rules, see [24] and references therein.

The quadrature issue motivated the idea of taking advantage of the high regularity of $\operatorname{IgA}$ basis functions to construct efficient and geometrically flexible collocation methods, see [2] or the recent survey [41]. They will be discussed in the next section. 


\subsection{Isogeometric collocation methods}

The collocation approach to approximate the solution of (22) is based on the strong form of the problem: we look for $u_{h}^{C} \in \mathbb{V}_{h, 0}$ (see (25)) as the solution of

$$
\mathcal{L} u_{h}^{C}\left(\tau_{i}\right)=f\left(\tau_{i}\right), \quad i=1, \ldots, n_{h},
$$

where $\tau_{i} \in \Omega$ are the so-called collocation points. Writing $u_{h}^{C}=\sum_{i=1}^{n_{h}} c_{i} \varphi_{i}$, condition (30) gives rise to a linear system $A \mathbf{c}=\mathbf{F}$.

Isogeometric collocation emanates from the combination of the isogeometric technology and the standard collocation method. Following the isoparametric approach, we consider the approximation spaces $\mathbb{V}_{h, 0}$ and $\mathbb{V}_{h}$ spanned by the functions in (29), and we select the collocation points as

$$
\tau_{i}:=\mathbf{G}\left(\hat{\tau}_{i}\right), \quad i=1, \ldots, n_{h},
$$

where $\hat{\tau}_{i}$ are suitable points belonging to the parametric domain $\widehat{\Omega}$, see Figure 5 .

Usually, in collocation $\operatorname{IgA}$, the functions $\hat{\varphi}_{i}$ in (28) are chosen to be tensorproduct B-splines or NURBS. The major advantage of isogeometric collocation over Galerkin-type methods is the minimal computational effort with respect to quadrature, since for each degree of freedom only one point evaluation at the collocation point is required. This property leads to extremely easy and fast constructions of the corresponding linear systems.

On the other hand, in contrast to isogeometric Galerkin methods, the theoretical understanding of isogeometric collocation methods is not so mature yet. The optimal choice of the collocation points, the unisolvency of the resulting system and the accuracy performance of the obtained approximate solution are still main open issues. Some results in this direction are known in the one-dimensional setting for a special choice of the points $\hat{\tau}_{i}$ in (31), the so-called Tchebycheff-Demko points, see [2]. However, in practical applications, the most popular choice of $\hat{\tau}_{i}$ are the so-called Greville points corresponding to the selected spline space. In case of the polynomial spline space $\mathbb{S}_{p, \Xi}$, they can be computed as knot averages,

$$
\frac{\xi_{i+1}+\cdots+\xi_{i+p}}{p}
$$

and they are tensor-product variations in case of tensor-product spline spaces.

In this paper we focus on isogeometric collocation methods based on tensorproduct GB-splines. The above-mentioned Greville and Tchebycheff-Demko points can be defined for generalized spline spaces (if $p \geq 3$ ) in a similar way as for polynomial spline spaces. The latter points can be computed by a similar iterative algorithm as the one proposed for B-splines in [5, Chapter XIII], see [35]. Nevertheless, in our numerical experiments we take the collocation points as (the images of) the Greville points of the standard B-splines of the same degree. This choice greatly simplifies the computation without sacrificing any accuracy [35]. 


\section{Examples}

In this section we illustrate the use of GB-splines as basis functions (28) in isogeometric Galerkin and collocation methods. We will show that a proper selection of the section spaces (1) according to a problem-dependent strategy may result in an accuracy gain compared with standard B-splines. To this end, we present three examples. The first one is an eigenvalue problem for the one-dimensional Laplace operator. In the second example we address an advection-dominated advection-diffusion problem on the unit square. Finally, in our third example we consider a vector problem (linear elasticity) on a bivariate domain $\Omega$ whose description requires a non-trivial geometry map. In the first and last example we will use a Galerkin discretization while for the advection-diffusion problem the isogeometric collocation approach is considered.

\subsection{An eigenvalue problem}

In our first example we consider the following eigenvalue problem for the univariate Laplace operator:

$$
\left\{\begin{array}{l}
-D^{2} u(x)=\omega^{2} u(x), \quad x \in(0,1), \\
u(0)=0, \quad u(1)=0,
\end{array}\right.
$$

whose non-trivial exact solutions are

$$
u_{k}(x):=\sin \left(\omega_{k} x\right), \quad \omega_{k}:=k \pi, \quad k=1,2, \ldots
$$

The weak form of problem (32) reads as follows: find non-trivial $u \in H_{0}^{1}(0,1)$ and $\omega$ such that

$$
\int_{0}^{1} D u(s) D v(s) \mathrm{d} s-\omega^{2} \int_{0}^{1} u(s) v(s) \mathrm{d} s=0, \quad \forall v \in H_{0}^{1}(0,1) .
$$

Following the Galerkin approach, we choose a subspace $\mathbb{V}_{h, 0}$ of $H_{0}^{1}(0,1)$ spanned by the basis $\left\{\varphi_{1}, \ldots, \varphi_{n_{h}}\right\}$ and we find approximate values $\omega_{h}^{G}$ to $\omega$ by solving

$$
K \mathbf{u}=\left(\omega_{h}^{G}\right)^{2} M \mathbf{u}
$$

where the matrices $K$ and $M$ consist of the elements

$$
K_{i, j}:=\int_{0}^{1} D \varphi_{j}(s) D \varphi_{i}(s) \mathrm{d} s, \quad M_{i, j}:=\int_{0}^{1} \varphi_{j}(s) \varphi_{i}(s) \mathrm{d} s, \quad i, j=1, \ldots, n_{h} .
$$

This means that each $\left(\omega_{h}^{G}\right)^{2}$ is an eigenvalue of the matrix $L:=M^{-1} K$. Thus, the $n_{h}$ eigenvalues of the matrix $L$ are an approximation of the first $n_{h}$ eigenvalues of the problem (32), namely 


$$
\omega_{k}^{2}=(k \pi)^{2}, \quad k=1, \ldots, n_{h} .
$$

Similarly, an approximation of the eigenfunction $u_{k}$ is obtained by considering

$$
\sum_{i=1}^{n_{h}} u_{k, i} \varphi_{i}
$$

where $\mathbf{u}_{k}:=\left(u_{k, 1}, \ldots, u_{k, n_{h}}\right)$ is the $k$-th eigenvector of $L$. Of course, a proper normalization is needed. Taking into account the expression of $u_{k}$ in (33), we normalize $\mathbf{u}_{k}$ so that the infinity-norm of the function in (34) is equal to 1 . More information on this eigenvalue problem can be found in [4].

We now choose the approximation space $\mathbb{V}_{h, 0}$ to be a space of smooth generalized spline functions vanishing at the two ends of the unit interval. More precisely, for $p \geq 2, n \geq 1$ we set

$$
\mathbb{V}_{h, 0}:=\left\{f \in \mathbb{S}_{n, p}^{U, V}: f(0)=f(1)=0\right\},
$$

where we recall $\mathbb{S}_{n, p}^{U, V}$ from (15). This space has dimension $n+p-2$, and is spanned by the set of GB-splines $\left\{B_{2, p}^{U, V}, \ldots, B_{n+p-1, p}^{U, V}\right\}$. Furthermore, we set

$$
\begin{aligned}
n K_{n, p}^{U, V} & :=\left[\int_{0}^{1} D B_{j+1, p}^{U, V}(s) D B_{i+1, p}^{U, V}(s) \mathrm{d} s\right]_{i, j=1}^{n+p-2}, \\
\frac{1}{n} M_{n, p}^{U, V} & :=\left[\int_{0}^{1} B_{j+1, p}^{U, V}(s) B_{i+1, p}^{U, V}(s) \mathrm{d} s\right]_{i, j=1}^{n+p-2}
\end{aligned}
$$

It is clear that the matrices $K_{n, p}^{U, V}$ and $M_{n, p}^{U, V}$ are symmetric, and due to the compact support of the GB-spline basis, they have a $(2 p+1)$-band structure. As discussed before, in view of the eigenvalue problem (32), we need to determine the eigenvalues of the matrix

$$
L_{n, p}^{U, V}:=n^{2}\left(M_{n, p}^{U, V}\right)^{-1} K_{n, p}^{U, V} .
$$

Considering the form (33) of the solution, the natural choice of the section spaces for the GB-splines is (4) with parameter $n \alpha$. Note that the corresponding spaces $\mathbb{S}_{n, p}^{\mathbb{T}_{n \alpha}}$ are not nested for increasing values of $n$. We denote by $K_{n, p}^{\mathbb{T}_{n \alpha}}, M_{n, p}^{\mathbb{T}_{n \alpha}}$ the matrices in (35) and (36) obtained with such a choice. Taking into account (17), we see that the central part of the matrices $K_{n, p}^{\mathbb{T}_{n \alpha}}, M_{n, p}^{\mathbb{T}_{n \alpha}}$ has a Toeplitz structure. A Toeplitz matrix is a square matrix whose entries are constant along each diagonal. More precisely, for $k=0,1, \ldots, p$ and $i=2 p, \ldots, n-p-1$,

$$
\begin{array}{r}
\left(K_{n, p}^{\mathbb{T}_{n \alpha}}\right)_{i, i \pm k}=\int_{0}^{p+1} D \phi_{p}^{\mathbb{T}_{\alpha}}(t \mp k) D \phi_{p}^{\mathbb{T}_{\alpha}}(t) \mathrm{d} t, \\
\left(M_{n, p}^{\mathbb{T}_{n \alpha}}\right)_{i, i \pm k}=\int_{0}^{p+1} \phi_{p}^{\mathbb{T}_{\alpha}}(t \mp k) \phi_{p}^{\mathbb{T}_{\alpha}}(t) \mathrm{d} t .
\end{array}
$$


These central parts agree with Toeplitz matrices generated by the functions

$$
\begin{aligned}
& f_{p}^{\mathbb{T}_{\alpha}}(\theta):=\sum_{k=-p}^{p}\left(\int_{0}^{p+1} D \phi_{p}^{\mathbb{T}_{\alpha}}(t-k) D \phi_{p}^{\mathbb{T}_{\alpha}}(t) \mathrm{d} t\right) \cos (k \theta), \\
& h_{p}^{\mathbb{T}_{\alpha}}(\theta):=\sum_{k=-p}^{p}\left(\int_{0}^{p+1} \phi_{p}^{\mathbb{T}_{\alpha}}(t-k) \phi_{p}^{\mathbb{T}_{\alpha}}(t) \mathrm{d} t\right) \cos (k \theta),
\end{aligned}
$$

respectively, see [42]. These functions are defined on $[-\pi, \pi]$, but since they are symmetric we can restrict them to the range $[0, \pi]$ for visualization purposes. The selection of the parameter $\alpha$ is crucial in this approximation strategy, and we refer to [42] for a discussion of different selection criteria.

For the sake of comparison, we also consider the Galerkin approximation of the solution of (33) by using standard B-splines of degree $p$ over the same knot sequence (13). The resulting matrices (35) and (36) have the same structure as $K_{n, p}^{\mathbb{T}_{n \alpha}}$ and $M_{n, p}^{\mathbb{T}_{n \alpha}}$. In particular, their central parts are Toeplitz matrices generated by the functions

$$
\begin{aligned}
& f_{p}(\theta):=\sum_{k=-p}^{p}\left(\int_{0}^{p+1} D \phi_{p}(t-k) D \phi_{p}(t) \mathrm{d} t\right) \cos (k \theta), \\
& h_{p}(\theta):=\sum_{k=-p}^{p}\left(\int_{0}^{p+1} \phi_{p}(t-k) \phi_{p}(t) \mathrm{d} t\right) \cos (k \theta),
\end{aligned}
$$

respectively, see [20].

In Figure 6 we plot the relative spectral error values

$$
\left(\frac{k}{n},\left(\frac{\omega_{k}^{\mathbb{T}_{n \alpha}}}{k \pi}\right)^{2}-1\right), \quad k=1, \ldots, n,
$$

where $\omega_{k}^{\mathbb{T} n} n$ denotes the trigonometric GB-spline approximation for $\omega_{k}$. In the same figure we also show the graph of the function

$$
e_{p}^{\mathbb{T}_{\alpha}}(\theta):=\frac{f_{p}^{\mathbb{T}_{\alpha}}(\theta)}{h_{p}^{\mathbb{T}_{\alpha}}(\theta)} \frac{1}{\theta^{2}}-1,
$$

which gives an asymptotic description of the quantity in (38), see [42]. For comparison, we also plot the relative spectral error values and the graph of the corresponding function $e_{p}$ in case of standard B-splines of the same degree over the same knot sequence, i.e.,

$$
e_{p}(\theta):=\frac{f_{p}(\theta)}{h_{p}(\theta)} \frac{1}{\theta^{2}}-1
$$



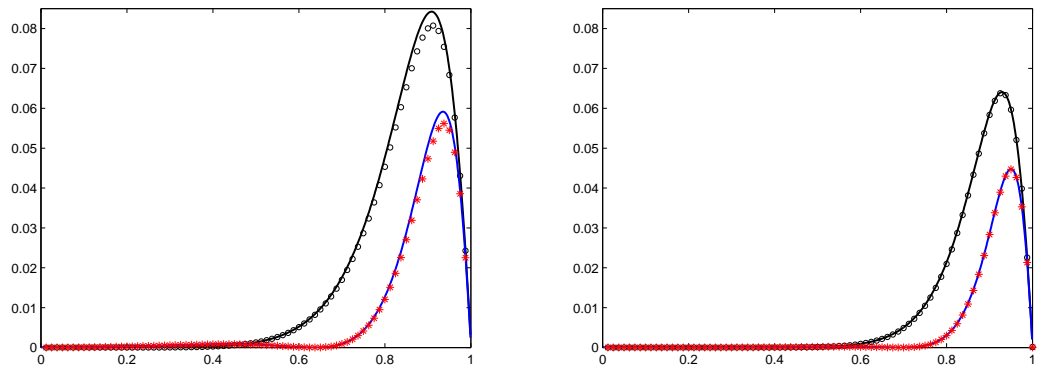

Fig. 6 Eigenvalue problem. Plot of the relative spectral error values in the trigonometric case $\left(\right.$ red $*$ ) and the polynomial case (black $\circ$ ) for $n=80$, together with the scaled graphs of $e_{p}^{\mathbb{T}_{\alpha}}$ (blue line) and $e_{p}$ (black line). Left: $p=3, \alpha=\frac{7}{11} \pi$. Right: $p=4, \alpha=\frac{9}{13} \pi$.
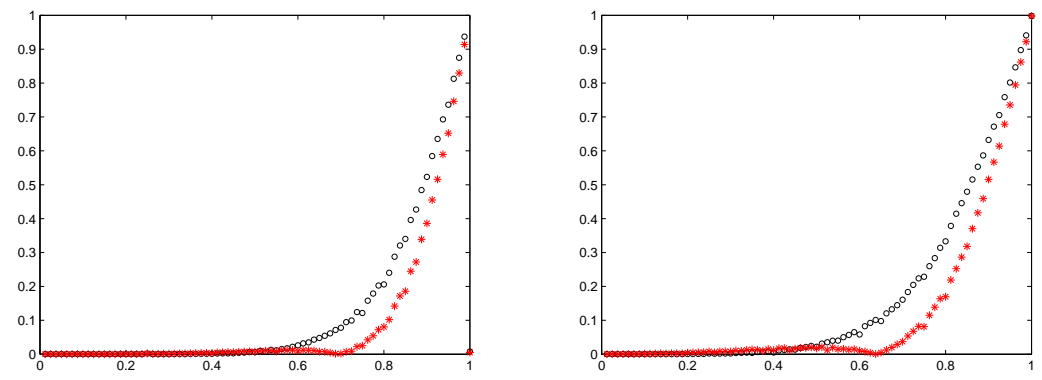

Fig. 7 Eigenvalue problem. Plot of the infinity-norm of the error for all the eigenfunctions in the trigonometric case (red $*$ ) and the polynomial case (black $\circ$ ) for $n=80$. Left: $p=3, \alpha=\frac{7}{11} \pi$. Right: $p=4, \alpha=\frac{9}{13} \pi$.

Both the functions are rescaled over the interval $[0,1]$ in the figure. The values of $\alpha$ are selected according to the heuristic suggestion ${ }^{1}$ in [35, Eq. (18)]. Figure 7 depicts the infinity-norm of the error for all the obtained eigenfunctions tabulated at 500 equally spaced points in $[0,1]$.

Two remarks are in order. First, there is a very good match between the functions $e_{p}^{\mathbb{T}_{\alpha}}$ obtained by the theoretical spectral analysis of the involved matrices and the computed relative error in (38) of the Galerkin approximation of the spectrum based on trigonometric spline spaces $\mathbb{S}_{n, p}^{\mathbb{T}_{n \alpha}}$. The same holds for the approximation based on standard B-splines. Second, trigonometric GB-splines present improvements over standard B-splines in the global approximation of both the eigenvalues and the eigenfunctions.

${ }^{1}$ For polynomial B-splines, a degree $p$ in Galerkin approximation corresponds to a degree $2 p+1$ in collocation, see [17, Remark 3.2]. We have followed the same rule here. 

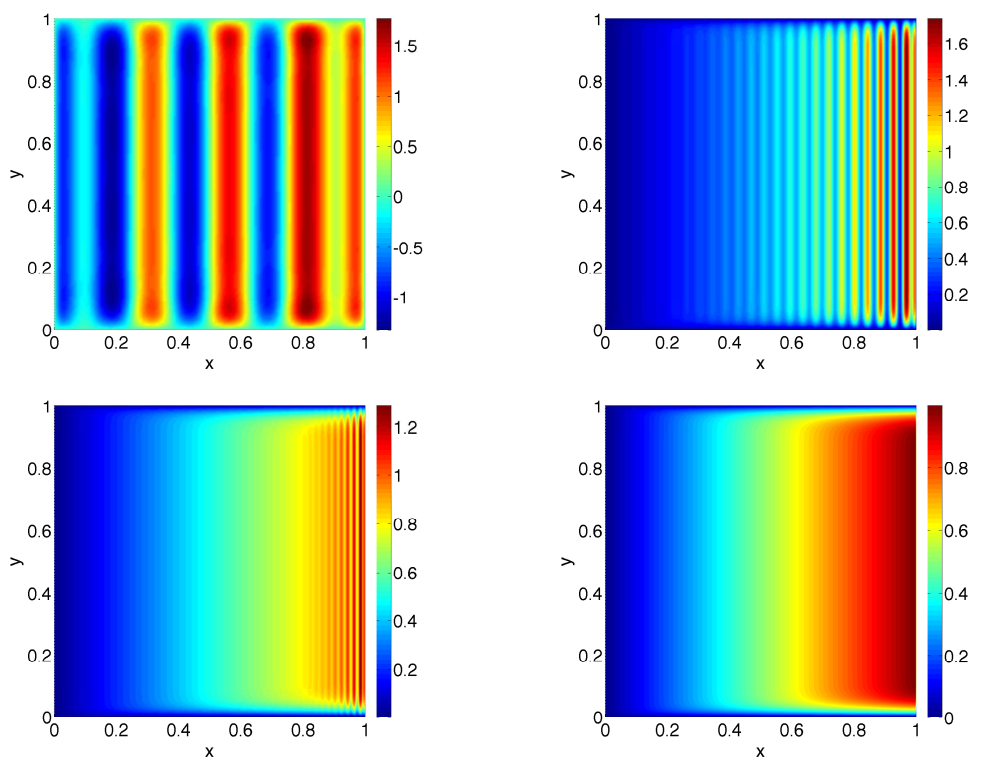

Fig. 8 Advection-diffusion problem. Contour plots of the numerical solution. Collocation is performed at standard tensor-product B-spline Greville points, using degree $p=4$ in both directions and standard tensor-product B-splines (top, left: $10 \times 10$ degrees of freedom; top, right: $50 \times 50$; bottom, left: $100 \times 100$; bottom, right: $300 \times 300$ ).

\subsection{An advection-diffusion problem with boundary layers}

In our second example we consider the following advection-diffusion problem defined on the bivariate domain $\Omega=(0,1)^{2}$ :

$$
-\kappa \Delta u+\boldsymbol{\beta} \cdot \nabla u=f, \quad \text { in } \Omega,
$$

complemented by homogeneous Dirichlet boundary conditions. The parameters are assumed to be $\kappa=10^{-3}$ and $\beta=\left[\begin{array}{ll}1 & 0\end{array}\right]^{T}$, while the right-hand side has a constant value $f=1$. The exact solution of such a problem is a ramp of unit slope along the $x$-axis, showing two layers at $y=0$ and $y=1$, and a third, sharper layer at $x=1$.

The solution of the problem is approximated by collocation IgA considering both tensor-product polynomial B-splines and tensor-product GB-splines. The physical domain and the parametric domain agree so there is no need of a geometry map. In both cases we use standard tensor-product B-spline Greville points as collocation points and we consider uniform knot sequences as in (13). Since the solution possess strong gradients and thin boundary layers it is natural to use the section spaces for our GB-splines as in (3), that is to use hyperbolic GB-splines.

We choose the parameter $\alpha$ to be equal to the global Péclet number, i.e., $\alpha=$ $\|\beta\| / \kappa=10^{3}$. In Figures 8 and 9 we present some results for the same degree $p=4$ in 

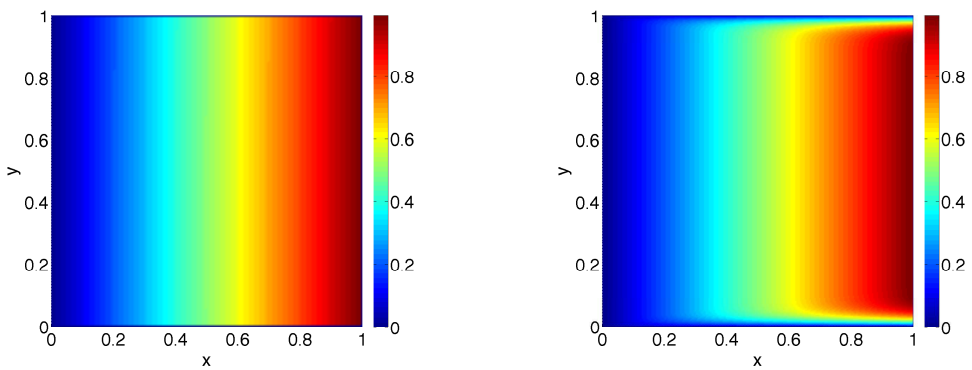

Fig. 9 Advection-diffusion problem. Contour plots of the numerical solution. Collocation is performed at standard tensor-product B-spline Greville points, using degree $p=4$ in both directions and tensor-product hyperbolic B-splines with $\alpha=\|\boldsymbol{\beta}\| / \kappa=10^{3}$ (left: $10 \times 10$ degrees of freedom; right: $50 \times 50$ ).

both the directions and different choices of uniform knot sequences. We can see that, as expected, standard B-splines lead to spurious oscillations until the discretization is fine enough to resolve the boundary layers. A discretization consisting of $300 \times$ 300 degrees of freedom $(n=298)$ is needed to get a solution where the oscillations (indeed still present) are negligible. Instead, with hyperbolic GB-splines and the adopted selection of the parameter $\alpha$, already a very coarse discretization $(10 \times 10$ degrees of freedom) leads to acceptable results. However, we notice that in this case the two boundary layers at $y=0$ and $y=1$ appear to be too sharp, as compared with the behavior of the $300 \times 300 \mathrm{~B}$-spline case. A less coarse discretization $(50 \times 50$ degrees of freedom) gives a solution that, in practice, cannot be distinguished from the $300 \times 300 \mathrm{~B}$-spline case. Finally, we remark that, in contrast to the $300 \times 300 \mathrm{~B}-$ spline discretization, no oscillations (not even very small) are observed in this case. Other advection-diffusion problems discretized by isogeometric collocation using GB-splines can be found in [35].

\subsection{A problem in solid mechanics}

In our last example we address a model problem in solid mechanics. We consider an infinite plate with a circular hole of radius $r$, subject to an in-plane uniform tension $T_{x}$ in $x$-direction, see Figure 10 (left). For a homogeneous and isotropic material this problem features an exact solution which can be found in [23, Section 7.6]. The infinite plate is modeled by a finite circular domain with radius $R$. Due to the symmetry, the computational domain $\Omega$ is restricted to a quarter, see Figure 10 (right). We study the linear elastic behavior of the displacement field $\mathbf{u}: \Omega \rightarrow \mathbb{R}^{2}$ described by

$$
\operatorname{div} \sigma(\mathbf{u})=0, \quad \text { in } \Omega .
$$



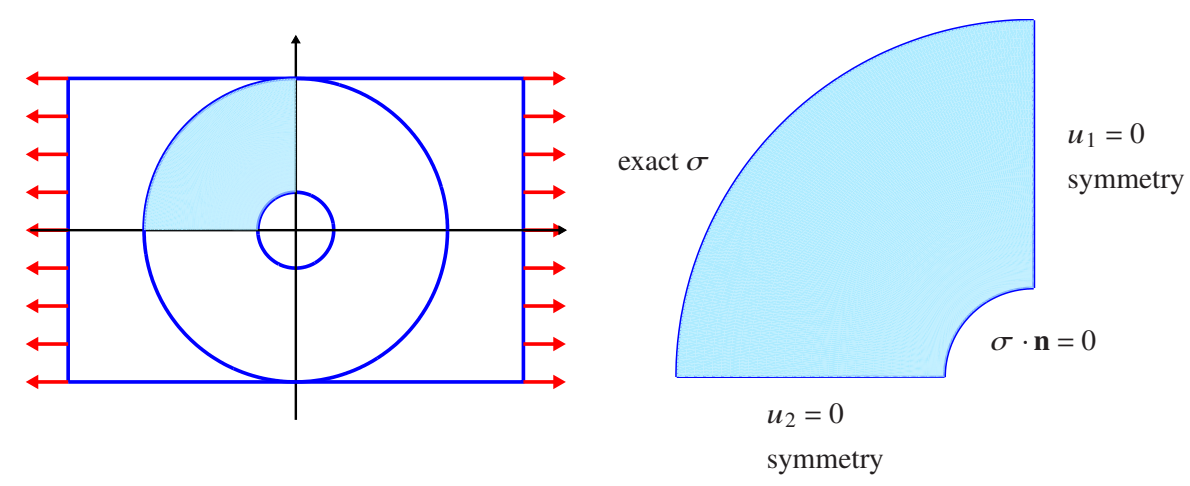

Fig. 10 Elastic plate problem with a circular hole.

The boundary conditions are shown in Figure 10 (right) where we apply the exact solution as Neumann boundary condition. For the sake of completeness, we recall that $\sigma(\mathbf{u}):=\left\{\sigma_{i j}(\mathbf{u})\right\}_{i, j=1,2}$ with

$$
\sigma_{i j}(\mathbf{u}):=\lambda \operatorname{div} \mathbf{u} \delta_{i j}+2 \mu \epsilon_{i j}(\mathbf{u}), \quad \epsilon_{i j}(\mathbf{u}):=\frac{1}{2}\left(\frac{\partial u_{i}}{\partial x_{j}}+\frac{\partial u_{j}}{\partial x_{i}}\right), \quad i, j=1,2,
$$

and

$$
\mathbf{u}:=\left(u_{1}, u_{2}\right), \quad(x, y):=\left(x_{1}, x_{2}\right), \quad \lambda:=\frac{E v}{(1+v)(1-2 v)}, \quad \mu:=\frac{E}{2(1+v)},
$$

where $E$ denotes the Young modulus and $v$ the Poisson ratio. In our computed example we have taken

$$
r=1, \quad R=4, \quad E=10^{5}, \quad v=0.3, \quad T_{x}=10 .
$$

Without the hole, the stress would be uniform

$$
\sigma_{1,1}=T_{x}, \quad \sigma_{1,2}=\sigma_{2,2}=0
$$

This distribution will alter only in the vicinity of the hole. More precisely, we get a peak stress concentration at the upper side of the hole, that is at the point $(0,1)$, see [23]. Due to the behavior of the solution, a numerical approximation strategy using local refinement around this point is preferable.

The solution of the problem is approximated by Galerkin IgA. We refer to [40, Chapter 3] for the weak form of the problem. Since the physical domain $\Omega$ is a quarter of an annulus, see Figure 10 (right), it is natural to use (quadratic) trigonometric GB-splines for the representation of the circular arcs. Hence, we construct a global geometry function as in (27) considering the tensor-product space $\mathbb{S}_{n, 2}^{\mathbb{T}} \otimes_{n, 2}$ with $\alpha=\frac{\pi}{2}$. The control points $\mathbf{P}_{i}=\mathbf{P}_{k, j}, k, j=1,2,3$, are depicted in Table 1 for a coarse grid consisting of one interval per edge, see Figure 11. Then, we approximate both 


\begin{tabular}{|c|ccc|}
\hline$k$ & $\mathbf{P}_{k, 1}$ & $\mathbf{P}_{k, 2}$ & $\mathbf{P}_{k, 3}$ \\
\hline 1 & $(-1,0)$ & $(-2.5,0)$ & $(-4,0)$ \\
2 & $(-1,1)$ & $(-2.5,2.5)$ & $(-4,4)$ \\
3 & $(0,1)$ & $(0,2.5)$ & $(0,4)$ \\
\hline
\end{tabular}

Table 1 Elastic plate problem. Control points of the geometry function.
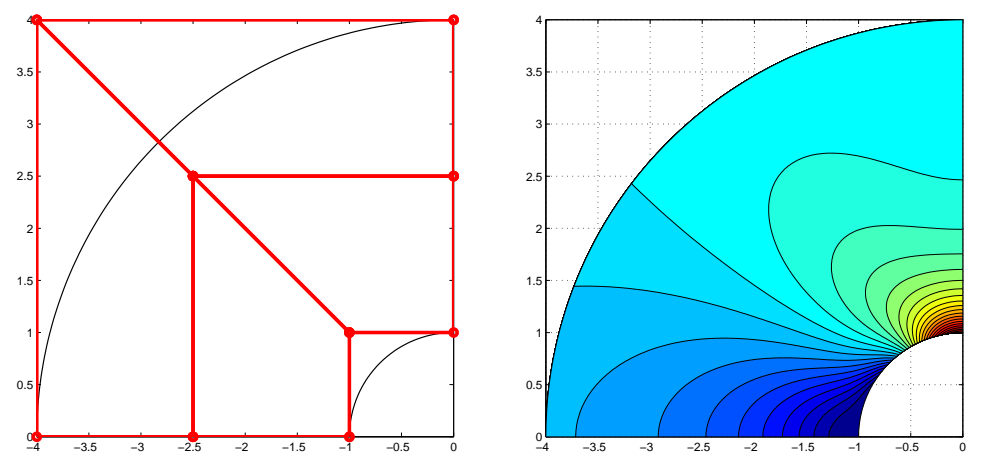

Fig. 11 Elastic plate problem. Left: control net of the geometry function. Right: exact $\sigma_{1,1}$.

components of the displacement in the bivariate tensor-product spaces $\mathbb{S}_{n, 2}^{\mathbb{T}_{\alpha}} \otimes \mathbb{S}_{n, 2}$ with $\alpha=\frac{\pi}{2}$ and different values of $n$. Standard quadratic B-splines $\mathbb{S}_{n, 2} \otimes \mathbb{S}_{n, 2}$ have also been considered for the sake of comparison. Next, we consider bivariate spaces spanned by hierarchical bases built from the same tensor-product spaces mentioned before, defined on the hierarchical meshes shown in Figure 12 (left column) according to the construction outlined in Section 2.4. Figure 13 shows the reduction of the computed error versus the number of degrees of freedom, in the cases of uniform and local hierarchical refinements with the different considered spaces. The presented hierarchical meshes are constructed manually. For automatic refinement strategies we refer to the literature, see e.g. [3, 18]. Further details on this example can be found in [34].

\section{Conclusions}

In this paper we have reviewed the definition and main properties of GB-splines, and we have illustrated their use in isogeometric Galerkin and collocation methods as a possible alternative to standard B-splines/NURBS.

GB-splines are a special case of the larger and richer class of Tchebycheffian Bsplines, and they can be seen as the minimal extension of the standard polynomial B-splines sharing similar properties. In particular, GB-splines support local refinement based on the hierarchical/multilevel approach. The complete structural simi- 

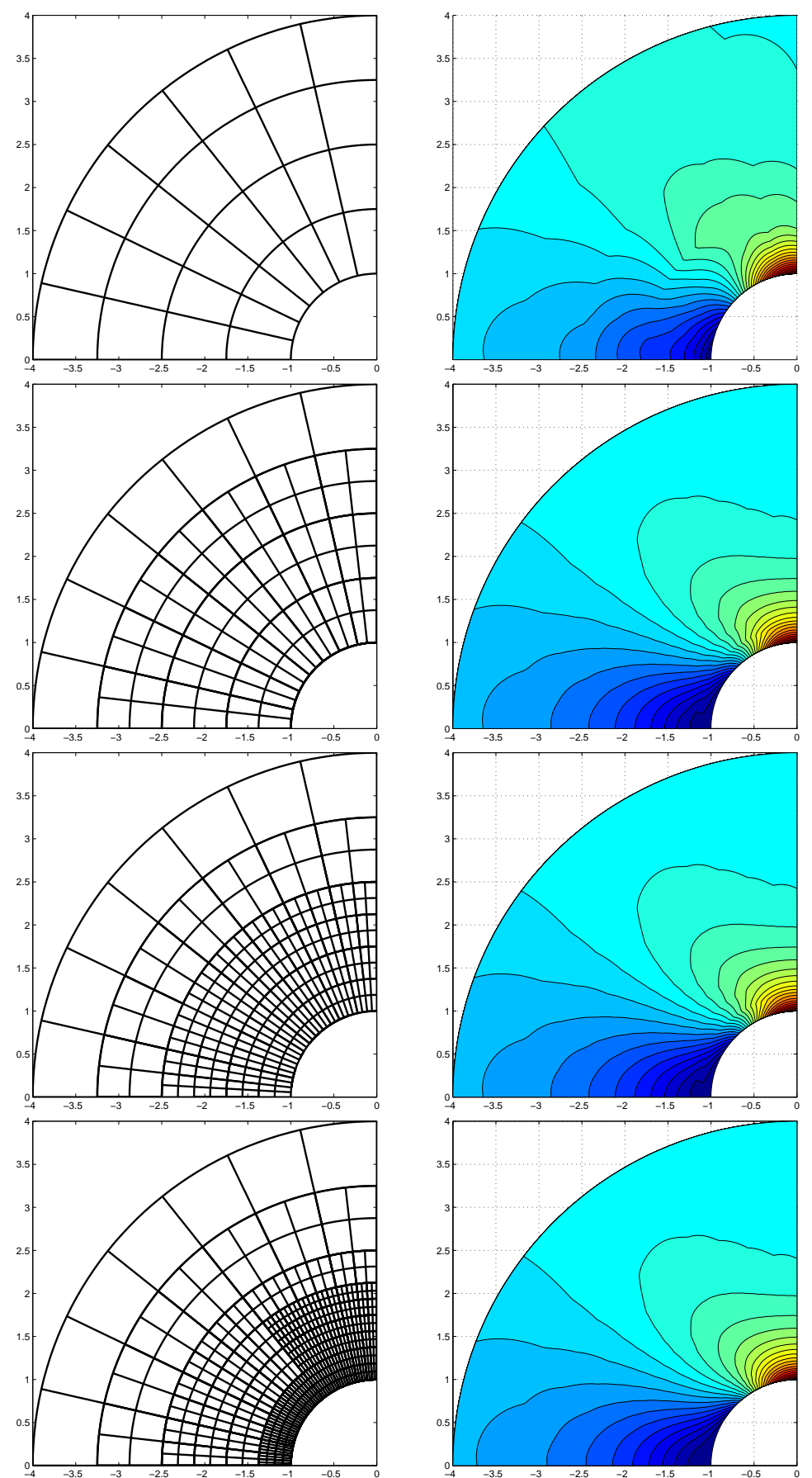

Fig. 12 Elastic plate problem. Hierarchical meshes and corresponding hierarchical quadratic trigonometric GB-spline approximations of $\sigma_{1,1}$ with $\alpha=\pi / 2$. 


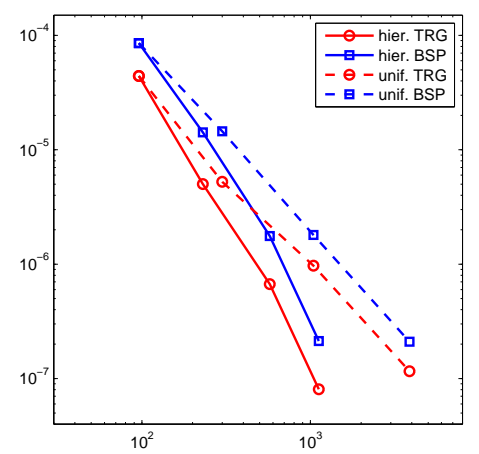

Fig. 13 Elastic plate problem. $L_{2}$-norm of the error for the displacement using quadratic trigonometric GB-splines (red line) and standard quadratic B-splines (blue line) versus the number of degrees of freedom, computed on locally refined hierarchical meshes (solid) and uniformly refined meshes (dashed).

larity between B-splines and GB-splines makes them plug-to-plug in isogeometric Galerkin and collocation methods.

Trigonometric and hyperbolic GB-splines are of particular interest in IgA because they offer some relevant advantages over NURBS whenever the section spaces are selected according to a problem-dependent strategy. In particular, trigonometric/hyperbolic GB-splines

- allow for the exact representation of conics by means of a parameterization which is close to the arc length, without requiring rational forms;

- behave completely similar to standard B-splines with respect to differentiation/integration;

- give rise to matrices with spectral properties completely analogous to standard B-splines when used in Galerkin/collocation methods;

- have the same approximation properties as standard B-splines of the same degree, for fixed values of the local phase parameters $\alpha$;

- approach standard B-splines of the same degree as the local phase parameters (or equivalently the knot spacing) approach zero.

Finally, we point out that GB-splines are also a promising tool in the context of Isogeometric Boundary Element Methods (IBEM), because of the advantages they offer for representing conics, see [1].

Acknowledgements This work was partially supported by INdAM Gruppo Nazionale per il Calcolo Scientifico and by the MIUR "Futuro in Ricerca 2013" Programme through the project "DREAMS". 


\section{References}

1. Aimi, A., Diligenti, M., Sampoli, M.L., Sestini, A.: Non-polynomial spline alternatives in isogeometric symmetric Galerkin BEM. Appl. Numer. Math. (in press) doi: 10.1016/j.apnum.2016.07.004

2. Auricchio, F., Beirão da Veiga, L., Hughes, T.J.R., Reali, A., Sangalli, G.: Isogeometric collocation methods. Math. Models Methods Appl. Sci. 20, 2075-2107 (2010).

3. Bank, R.E., Smith, R.K.: A posteriori error estimates based on hierarchical bases. SIAM J. Numer. Anal. 30, 921-935 (1993).

4. Boffi, D.: Finite element approximation of eigenvalue problems. Acta Numer. 19, 1-120 (2010).

5. de Boor, C.: A Practical Guide to Splines. Revised Edition. Springer, New York (2001).

6. Bracco, C., Berdinsky, D., Cho, D., Oh, M.-J., Kim, T.-W.: Trigonometric generalized Tsplines. Comput. Methods Appl. Mech. Engrg. 268, 540-556 (2014).

7. Bracco, C., Cho, D.: Generalized T-splines and VMCR T-meshes. Comput. Methods Appl. Mech. Engrg. 280, 176-196 (2014).

8. Bracco, C. Lyche, T., Manni, C., Roman, F., Speleers, H.: Generalized spline spaces over Tmeshes: Dimension formula and locally refined generalized B-splines. Appl. Math. Comput. 272, 187-198 (2016).

9. Bracco, C. Lyche, T., Manni, C., Roman, F., Speleers, H.: On the dimension of Tchebycheffian spline spaces over planar T-meshes. Comput. Aided Geom. Design 45, 151-173 (2016).

10. Bracco, C. Roman, F.: Spaces of generalized splines over T-meshes. J. Comput. Appl. Math. 294, 102-123 (2016).

11. Carnicer, J.M., Mainar, E., Peña, J.M.: Critical length for design purposes and extended Chebyshev spaces. Constr. Approx. 20, 55-71 (2004).

12. Costantini, P.: Curve and surface construction using variable degree polynomial splines. Comput. Aided Geom. Design 17, 419-446 (2000).

13. Costantini, P., Lyche, T., Manni, C.: On a class of weak Tchebycheff systems. Numer. Math. 101, 333-354 (2005).

14. Cottrell, J.A., Hughes, T.J.R., Bazilevs, Y.: Isogeometric Analysis: Toward Integration of CAD and FEA. John Wiley \& Sons, Chichester (2009).

15. Deng, J., Chen, F., Feng, Y.: Dimensions of spline spaces over T-meshes. J. Comput. Appl. Math. 194, 267-283 (2006).

16. Dokken, T., Lyche, T., Pettersen, K.F.: Polynomial splines over locally refined box-partitions. Comput. Aided Geom. Design 30, 331-356 (2013).

17. Donatelli, M., Garoni, C., Manni, C., Serra-Capizzano, S., Speleers, H.: Spectral analysis and spectral symbol of matrices in isogeometric collocation methods. Math. Comp. 85, 16391680 (2016).

18. Dörfel, M., Jüttler, B., Simeon, B.: Adaptive isogeometric analysis by local $h$-refinement with T-splines. Comput. Methods Appl. Mech. Engrg. 199, 264-275 (2010).

19. Forsey, D.R., Bartels, R.H.: Hierarchical B-spline refinement. Comput. Graph. 22, 205-212 (1988).

20. Garoni, C., Manni, C., Pelosi, F., Serra-Capizzano, S., Speleers, H.: On the spectrum of stiffness matrices arising from isogeometric analysis. Numer. Math. 127, 751-799 (2014).

21. Giannelli, C., Jüttler, B., Speleers, H.: THB-splines: The truncated basis for hierarchical splines. Comput. Aided Geom. Design 29, 485-498 (2012).

22. Giannelli, C., Jüttler, B., Speleers, H.: Strongly stable bases for adaptively refined multilevel spline spaces. Adv. Comp. Math. 40, 459-490 (2014).

23. Gould, P.L.: Introduction to Linear Elasticity. Springer-Verlag, Berlin (1999).

24. Hiemstra, R.R., Calabrò, F., Schillinger, D., Hughes, T.J.R.: Optimal and reduced quadrature rules for tensor product and hierarchically refined splines in isogeometric analysis. Comput. Methods Appl. Mech. Engrg. (in press) doi: 10.1016/j.cma.2016.10.049

25. Hughes, T.J.R., Cottrell, J.A., Bazilevs, Y.: Isogeometric analysis: CAD, finite elements, NURBS, exact geometry and mesh refinement. Comput. Methods Appl. Mech. Engrg. 194, 4135-4195 (2005). 
26. Jerome, J.W., Schumaker, L.L.: Local support bases for a class of spline functions. J. Approx. Theory 16, 16-27 (1976).

27. Koch, P.E., Lyche, T.: Interpolation with exponential B-splines in tension. In: Farin, G., Hagen, H., Noltemeier, H., Knödel, W. (eds.) Geometric Modelling, Springer-Verlag, 173-190 (1993).

28. Kvasov, B.I., Sattayatham, P.: GB-splines of arbitrary order. J. Comput. Appl. Math. 104, 63-88 (1999).

29. Li, X., Scott, M.A.: Analysis-suitable T-splines: Characterization, refineability, and approximation. Math. Models Methods Appl. Sci. 24, 1141-1164 (2014).

30. Lyche. L.: A recurrence relation for Chebyshevian B-splines. Constr. Approx. 1, 155-173 (1985).

31. Mainar, E., Peña, J.M., Sánchez-Reyes, J.: Shape preserving alternatives to the rational Bézier model. Comput. Aided Geom. Design 18, 37-60 (2001).

32. Manni, C., Pelosi, F., Sampoli, M.L.: Generalized B-splines as a tool in isogeometric analysis. Comput. Methods Appl. Mech. Engrg. 200, 867-881 (2011).

33. Manni, C., Pelosi, F., Sampoli, M.L.: Isogeometric analysis in advection-diffusion problems: Tension splines approximation. J. Comput. Appl. Math. 236, 511-528 (2011).

34. Manni, C., Pelosi, F., Speleers, H.: Local hierarchical $h$-refinements in IgA based on generalized B-splines. In: Floater, M., et al. (eds.) Mathematical Methods for Curves and Surfaces 2012, Lecture Notes in Computer Science 8177, 341-363 (2014).

35. Manni, C., Reali, A., Speleers, H.: Isogeometric collocation methods with generalized Bsplines. Comput. Math. Appl. 70, 1659-1675 (2015).

36. Manni, C., Speleers, H.: Standard and non-standard CAGD tools for isogeometric analysis: A tutorial. In: Buffa, A., Sangalli, G. (eds.) IsoGeometric Analysis: A New Paradigm in the Numerical Approximation of PDEs, Lecture Notes in Mathematics 2161, 1-69 (2016).

37. Mazure, M.L.: Four properties to characterize Chebyshev blossoms. Constr. Approx. 17, 319-334 (2001).

38. Mazure, M.L.: How to build all Chebyshevian spline spaces good for geometric design? Numer. Math. 119, 517-556 (2011).

39. Piegl, L., Tiller, W.: The NURBS Book (Monographs in Visual Communication). Second Edition. Springer-Verlag, New York (1997).

40. Quarteroni, A.: Numerical Models for Differential Problems. Springer-Verlag, Mailand (2009).

41. Reali, A., Hughes, T.J.R.: An introduction to isogeometric collocation methods. In: Beer, G., Bordas, S.P. (eds.) Isogeometric Methods for Numerical Simulation, Springer-Verlag, Wien (2015).

42. Roman, F., Manni, C., Speleers, H.: Spectral analysis of matrices in Galerkin methods based on generalized B-splines with high smoothness. Numer. Math. 135, 169-216 (2017).

43. Roman, F., Manni, C., Speleers, H.: Numerical approximation of GB-splines with a convolutional approach. Appl. Numer. Math. (in press) doi: 10.1016/j.apnum.2016.10.017

44. Schumaker, L.L.: Spline Functions: Basic Theory. Third Edition. Cambridge University Press, Cambridge (2007).

45. Schumaker, L.L., Wang, L.: Approximation power of polynomial splines on T-meshes. Comput. Aided Geom. Design 29, 599-612 (2012).

46. Sederberg, T., Zheng, J., Bakenov, A., Nasri, A.: T-splines and T-NURCCs. ACM Trans. Graphics 22, 477-484 (2003).

47. Speleers, H., Manni, C.: Effortless quasi-interpolation in hierarchical spaces. Numer. Math. 132, 155-184 (2016).

48. Wang, G., Fang, M.: Unified and extended form of three types of splines. J. Comput. Appl. Math. 216, 498-508 (2008). 